\title{
The 'Orochi' Flap Concept: Multi-Stage Combined Flap Using Sequential Flaps
}

\author{
Isao Koshima, $\mathrm{MD}^{1 *}$; Hirofumi Imai, MD; Shuhei Yoshida, MD; Takanobu Harima, MD²; \\ Haruki Mizuta, MD; Shuji Yamashita, MD²; Shogo Nagamatsu, MD; Kazunori Yokota, MD; \\ Ruben Kannan MD ${ }^{4}$
}

' International Center for Lymphedema, Hiroshima University Hospital, Hiroshima, Japan ${ }^{2}$ Department of Plastic and Reconstructive Surgery, Hiroshima University Hospital, Hiroshima, Japan ${ }^{3}$ Department of Plastic and Reconstructive Surgery, University of Tokyo Hospital, Tokyo, Japan

${ }^{4}$ Department of Plastic Surgery, Queen Victoria Hospital, East Grinstead, UK

\begin{abstract}
Flow-through flaps, wherein two or more flaps are placed in series using microvascular anastomoses, are indicated for the coverage of longer and wider defects, typically in extremities. These flaps are technically demanding but, more importantly, are associated with distal flap loss. To overcome these problems, the 'Orochi' concept of placing multiple flaps in parallel as part of a modular reconstruction has been put forward. In this article, this concept is illustrated in two cases of extremity reconstruction with the use of a sequential triple thoracodorsal perforator (TAP)-intercostal artery perforator (ICAP)-superficial circumflex iliac artery perforator (SCIP) mega-flap placed in parallel with a free TAP flap at the distal end of the defect. These modular mega-flaps are connected to a common vascular trunk by means of end-to-side microvascular anastomoses or perforator-to-perforator anastomoses to create parallel vascular systems; hence, the 'Orochi' (hydra-like) analogy. This allows for extensively long extremity defects of over $50 \mathrm{~cm}$ to be reconstructed safely. The use of the TAP-ICAP-SCIP model also confers the advantage of minimising operating time, negating the need for repeated positional change as with multiple flap elevation and a relatively concealed donor site.
\end{abstract}

\section{INTRODUCTION}

The reconstruction of longer and wider defects in extremities remains a challenge in the reconstructive setting. The extended latissimus dorsi musculocutaneous (LDMC) flap and anterolateral thigh (ALT) flap have longer vascular dimensions [1], but are still disadvantaged by the limited dimensions of a single angiosome and invasive donor sites. Hence, the need for lateral thinking to create a new flap or flap combinations with a longer reach alongside the reduced donor site morbidity. Sequential LDMC and groin flap or erstwhile named 'siamese' flaps had a great advantage for longer defects in the extremity. However, the major problems are (1) short pedicle length of the superficial circumflex iliac artery (SCIA) system, which restricts its longitudinal dimension, (2) positional change for flap elevation, and (3) requiring the sacrifice of the latissimus dorsi muscle.

To overcome the problems of sequential LDMC-groin flap without having to resort to flow-through flaps, we developed a combination of a free thoracodorsal artery perforator (TAP) and sequential TAP-intercostal artery perforator (ICAP)-superficial circumflex iliac artery perforator (SCIP) flaps. The latter used triple perforator flaps of the TAP, ICAP, and SCIP in sequence with additional supercharging. Unlike flow-through flaps, in which flaps are placed in series with a proportionate decrease in the hydrostatic pressure within the main vascular axis, the creation of parallel vascular pathways as shown in this article allows for the successful reconstruction of very long and narrow defects.

\section{SURGICAL TECHNIQUES}

In a retrospective study of two cases of extremity reconstruction, the 'Orochi' flap concept is illustrated as a multi-staged procedure. In the first stage, a free TAP flap is raised with the patient in the supine position, with a width of less than $8 \mathrm{~cm}$, allowing for direct closure. The TAP flap is then inset at the distal end of the defect with anend-to-side or perforator-to-perforator microvascular anastomosis, thereby creating a parallel vascular system. At the next stage, a sequential TAP-ICAP-SCIP triple mega-flap is raised using two concurrent surgical teams operating in the axillary and groin areas, respectively. Bloodless elevation is very important so as not to damage the perforators. For this purpose, the flap is elevated subfascially using cautery scissors, even around the perforators of the flap pedicle. The length of the SCIP flap's vascular pedicle can be increased by further dissecting the SCIA [2], allowing for greater ease of pivoting the flap. This manouevre necessitates the division of the lateral femoral cutaneous nerve (LCFN) as the SCIA system passes beneath it. The LCFN is subsequently coapted again after flap elevation [2].

As this mega-flap transverses three angiosomes, viz. the SCIP, TAP, and ICAP systems, the ICAP in the middle third of this sequential flap should also be dissected akin to the TAP and SCIP pedicles, and transected near the rib. This maximises the pedicle length. Its inclusion allows for an additional option of supercharging the mid-third of the mega-flap in case it is poorly vascularized. This may be needed if the ICAP system has hypoplastic or absent vascular channels. Once the flap is raised, it is pivoted on the TAP or SCIP pedicle, depending on whether the defect to be reconstructed is on the upper or lower extremity. Once inset, the distal flap vascular pedicle is supercharged in parallel to the main vessel via end-to-side or perforator-to-perforator anastomoses to further vascularise the mega-flap.

\section{CASE 1}

A 35-year-old carpenter developed necrotizing fascitis of a wide area of the left arm after a contaminated nail puncture injury. After radical debridement of soft tissue, the defect was initially reconstructed with a meshed split skin graft, which, however, caused severe scar contracture of the entire forearm and lateral portion of the upper arm, in the longer term (Figures $1 A, B$ ). In a subsequent surgery in the supine position, a free TAP flap $(35 \times 8 \mathrm{~cm})$ from the 

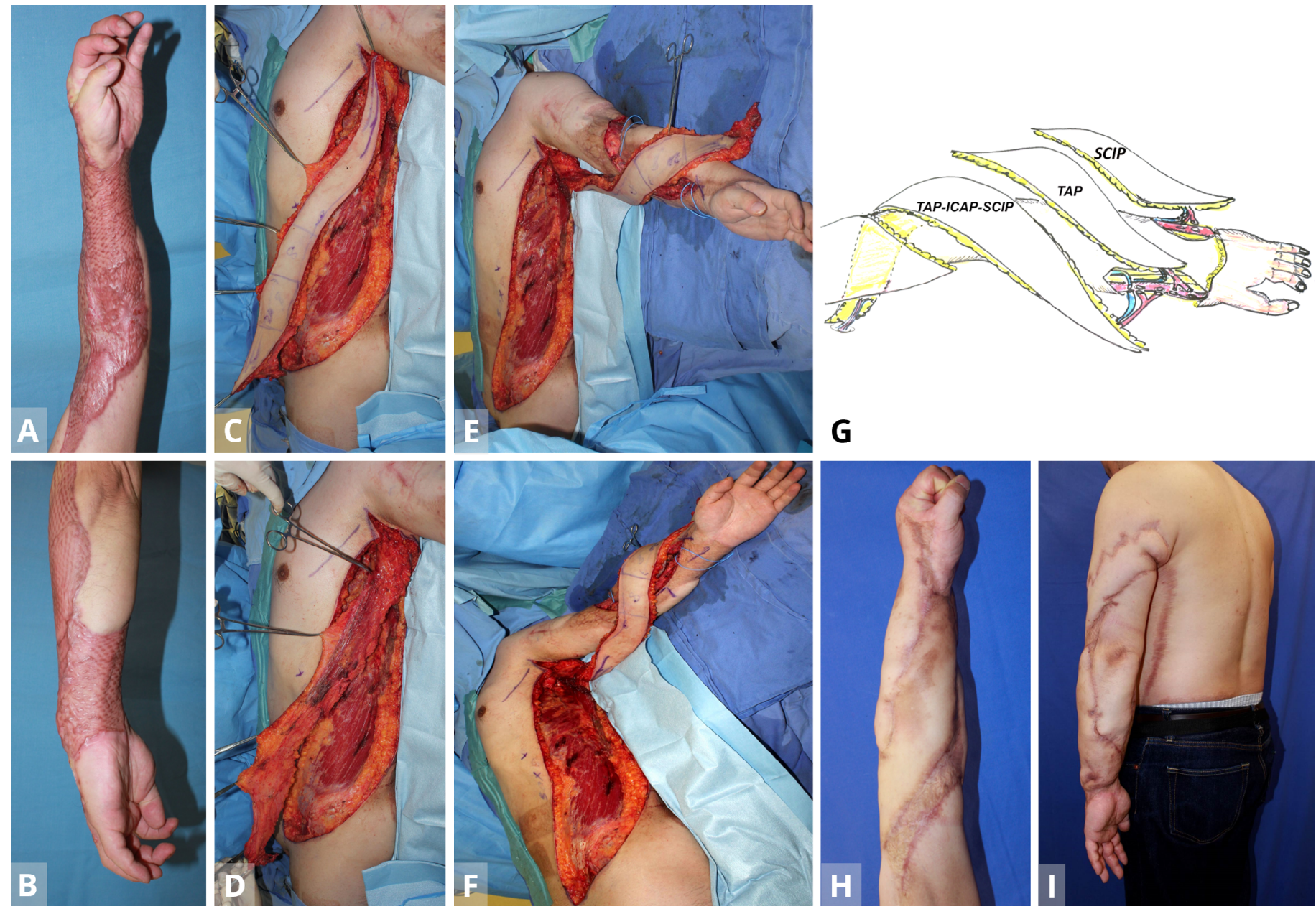

\section{G}
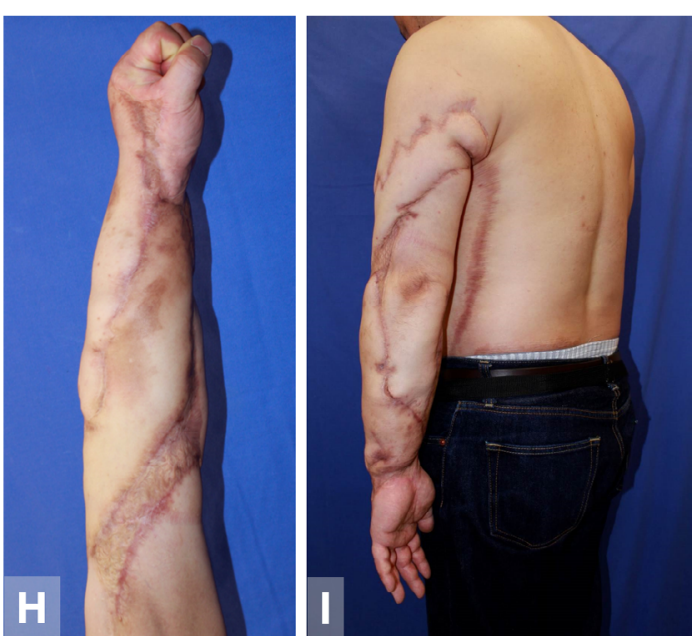

Figure 1. Case 1. (A,B) A 35-year old man with severe contracture due to mesh skin graft after wide necrotizing fascitis. The patient was unable to flex fingers. (C,D) After the primary free TAP flap is transferred from the contralateral lateral thoracic region for the dorsal forearm (stage 1), the TAP-ICAP-SCIP lap is elevated to reconstruct the volar forearm and upper arm defect (stage 2). (E,F) The triple TAP-ICAP-SCIP mega-flap in the second stage is raised and then inset to cover the more proximal spiral $50 \times 8 \mathrm{~cm}$ defect after releasing the contracture. (G) In the first stage, a free TAP flap from the contralateral middle axillary region is transferred to the posterior aspect of the forearm. Using end-to-side arterial anastomosis, the T-shaped pedicle of the TAP flap is anastomosed with the recipient radial vessel at the wrist level, creating a parallel vascular system to sustain the TAP flap. At the second stage, a sequential $50 \times 8 \mathrm{~cm}$ TAP-ICAP-SCIP mega-flap inset into the spiral defect from the proximal upper arm to the flexor side of the forearm. The SCIP component is distally supercharged by the recipient radial vessels at the level of the wrist (end-to-side) arterial anastomosis. At the third stage, contralateral SCIP flap is transferred to the ulnar aspect of the forearm. The superficial circumflex iliac artery and the cutaneous vein of SCIP flap are anastomosed to the recipient ulnar artery (end-to-side) and concomitant vein at the level of the wrist joint. $(\mathrm{H}, \mathrm{I})$ Normal power grip at five years later. The majority of the entire split skin graft is replaced with sequential triple TAP-ICAP-SCIP mega-flap, all in parallel. ICAP, intercostal artery perforator; SCIP, superficial circumflex iliac artery perforator; TAP, triple thoracodorsal perforator.

contralateral middle axillary region was transferred to release the contracture of the posterior aspect of the forearm and hand. Using end-to-side arterial anastomoses, the T-shaped pedicle of the TAP flap was anastomosed with the recipient radial vessel at the wrist level, creating a parallel vascular system to sustain the TAP flap. Donor site was closed directly.

At the second stage on June 25,2012, namely a year later, a sequential 50 $x 8 \mathrm{~cm}$ TAP-ICAP-SCIP mega-flap from the ipsilateral left side was raised, with the patient again in the supine position (Figures $1 C, D$ ). The flap was pivoted on the TAP pedicle, with the distal SCIA system of SCIP flap being transected to allow the flap to be pivoted and inset into the spiral defect. This allowed coverage of the defect from the proximal upper arm to the flexor side of the forearm. The SCIP component of the triple mega-flap was distally supercharged between the SCIA (artery) and the cutaneous vein of SCIP flap (vein) and the recipient radial vessels at the level of the wrist (end-to-side arterial anastomosis (Figures 1E-G). The donor defect was again closed primarily.

At the third stage, the contralateral SCIP flap was transferred to release contracture of the ulnar aspect of the forearm. The SCIA and the cutaneous vein of SCIP flap were anastomosed to the recipient ulnar artery (end-to-side) and concomitant vein (end-to-end) at the level of wrist joint (Figure 1G). Subsequently, the narrow skin area of these flaps was widened using silicone expanders (Figures $1 \mathrm{H}, \mathrm{I}$ ).

\section{CASE 2}

A 45-year-old woman had lipodystrophy over the anterior aspect of her left thigh as well as the medial aspect of the left knee (Figures 2A,B). As in Case 1 , the first stage entailed the use of the free TAP $(30 \times 7 \mathrm{~cm})$, in the supine position from the contralateral middle axillary region. The initial defect reconstructed was the distal-most part of the overall defect. Microvascular anastomosis was then performed with an end-to-end, perforator-to-perforator anastomosis between the flap pedicle and recipient perforators around the knee. Donor site was closed directly.

During the secondary surgery on March 22, 2013, a sequential TAP-ICAPSCIP flap $(50 \times 9 \mathrm{~cm})$ was again elevated from the ipsilateral left side in a spine position (Figures $2 \mathrm{C}, \mathrm{D}$ ). To cover a lower limb defect, the flap was based on the 


\section{INNOVATION}
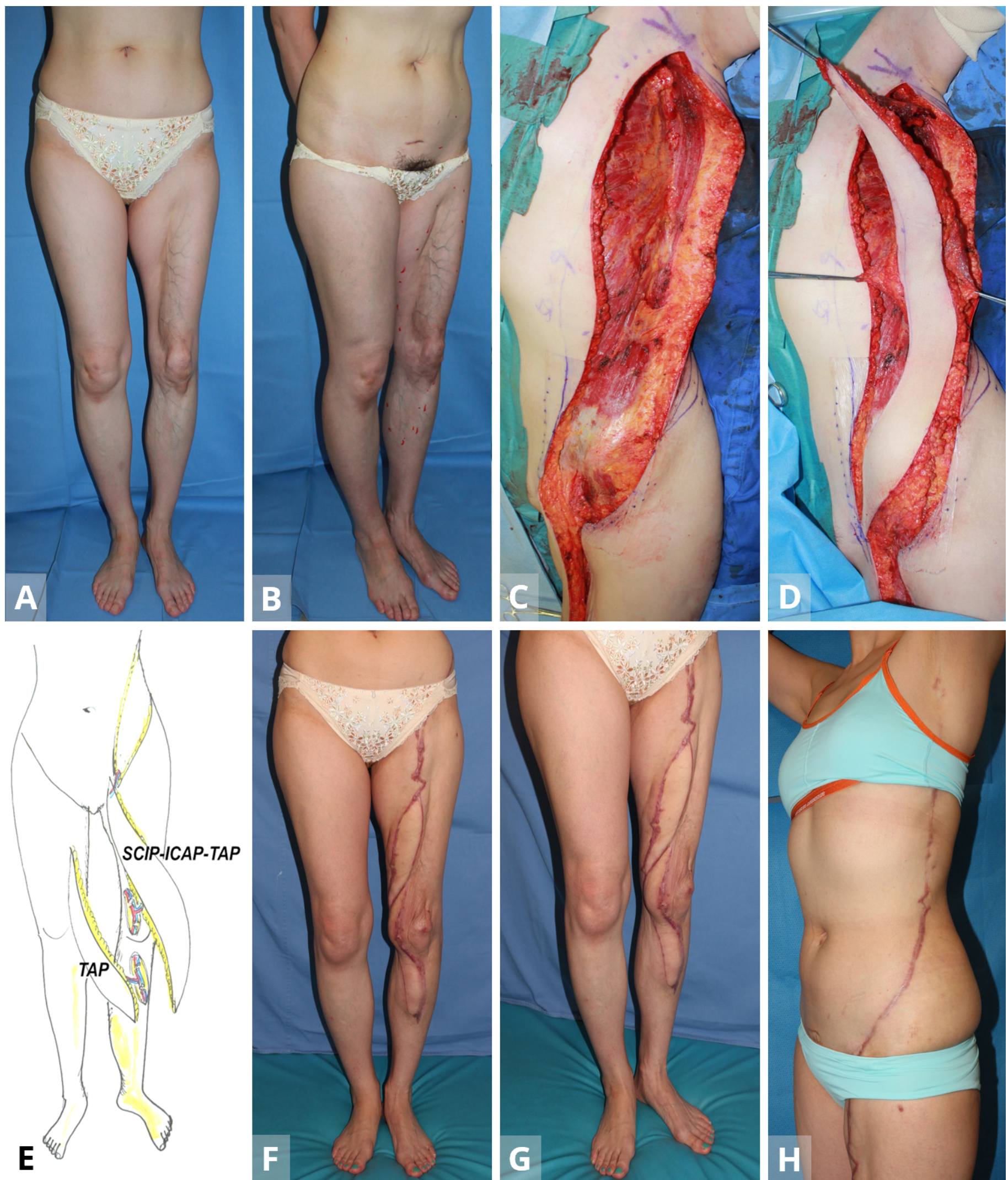

Figure 2. Case 2. (A,B) A 45-year-old woman with lipodystrophy on the anterior aspect on her left leg. (C,D) Following the primary TAP free-flap transfer from the contralateral side for lower leg, in the secondary surgery, the sequential TAP-ICAP-SCIP flap is elevated from the ipsilateral left side. (E) The first stage entails the use of the free TAP from the contralateral middle axillary region. The initial defect reconstructed is the distal-most part of the overall defect. During the secondary surgery, a sequential triple mega-flap mega flap ( $50 \times 9 \mathrm{~cm})$ based on the superficial circumflex iliac artery pedicle is pivoted into the longitudinal defect of the thigh. Supercharge of the distal pedicle is established between the transected TAP vessels and the perforators around the knee (recipient vessels) to create a parallel vascular system to the main vascular trunk of the lower limb. (F-H) Postoperative appearance at twenty months after completion of the 'Orochi' flap. ICAP, intercostal artery perforator; SCIP, superficial circumflex iliac artery perforator; TAP, triple thoracodorsal perforator. 
A

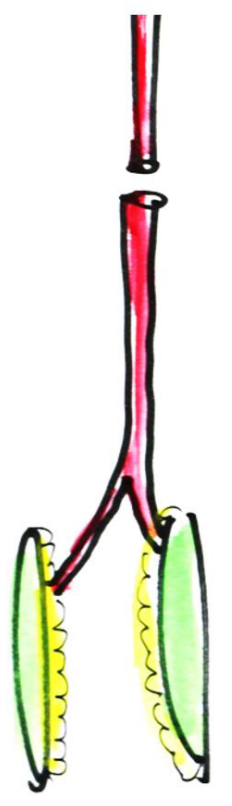

Hallock
B

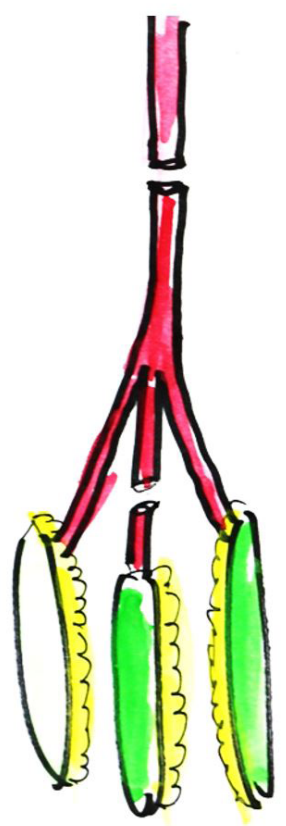

Koshima
C

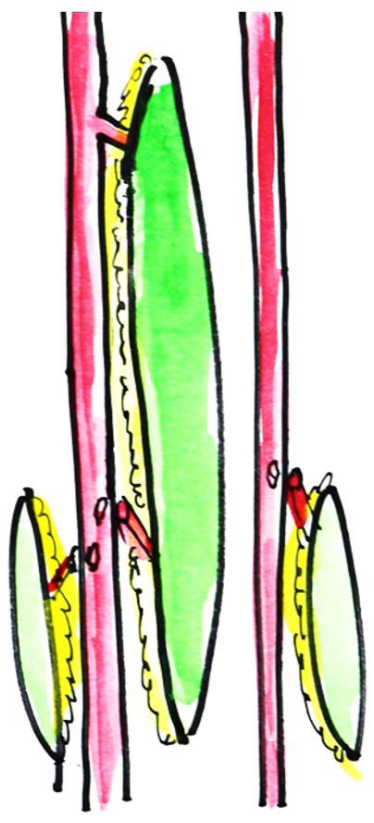

Case 1
D

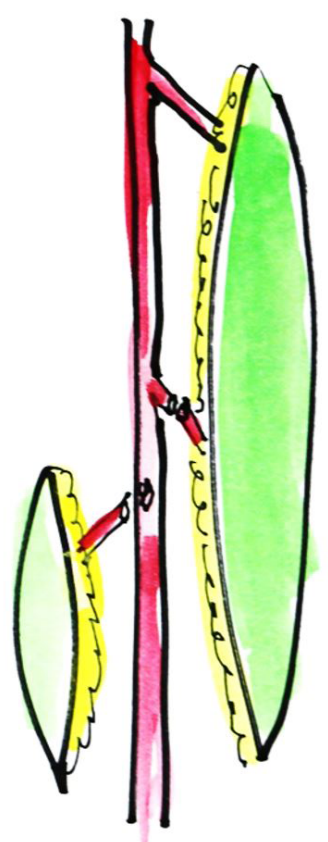

Case 2

Figure 3. Classification of free combined tissue transfer. (A) Classical chimera flap (Hallock). (B) Single stage chimera flap with additional free-flap based on a single recipient vessel (Koshima). (C) Triple stage ‘Orochi' flap. Triple mega-flap with double flaps based on double source vessels as seen in Case 1. (D) Two-stage ‘Orochi' flap. Triple mega-flap with a flap with a single source vessel as seen in Case 2 .

SCIA pedicle and pivoted into the longitudinal defect, proximal to the free TAP flap (stage 1). Supercharge of the distal pedicle was then established between the transected TAP vessels and the perforators around the knee (recipient vessels) via an end-to-end microvascular anastomosis to create a parallel vascular system to the main vascular trunk of the lower limb (Figure 2E). The donor site was closed primarily. Follow-up at twenty-months did not reveal any donor-site morbidity or functional disturbances (Figures $2 \mathrm{~F}-\mathrm{H}$ ).

In both of these cases, the postoperative course was smooth with no evidence of partial or total flap necrosis. Follow-up at five-years (Case 1) and twenty-months (Case 2) documented improvements in both functional and aesthetic terms, both in the donor and recipient areas (Figures 1G, 2E).

\section{DISCUSSION}

The reconstruction of very long and narrow defects, specifically of the extremities, has previously been described with the use of flaps such as the LDMC-groin flap [1], bilateral deep inferior epigastric artery perforator (DIEP) flaps [3-5], and the turbo-charged gracilis myocutaneous flap [6]. Others have similarly reported the Tensor Fasciae Latae (TFL)-sartorius musculocutaneous flap [7] as well as the ALT-SCIP flap [8]. These flaps, variously termed as 'siamese' or sequential flaps, however, are still relatively limited by their length. Sequential combinations of double flap transfers with double source vessels and distal vascular supercharge are known to provide sufficient nourishment for large skin territories. Transposing the perforator flap concept onto this allows for a more refined approach. In 2001, the authors summarized and classified the concept of combining tissue transfers $[9,10]$. Since that time, ad- ditional permutations and combinations of microsurgical options have been proposed. In particular, the perforator flap technology provides a less invasive procedure with reduced morbidity due to muscle preservation. It is also aesthetically superior to its predecessors, e.g., the LDMC-groin flap. This was the premise for the development of the triple TAP-ICAP-SCIP mega-flap for coverage of longer and wider defects in the extremities.

Hallock first reported the original chimera combined flap without an additional free-flap [11], while the authors expanded on this in their classification of chimeric flaps using a single source vessel [9,10,12-14] (Figure 3). The onestage chimeric combination of an anterolateral thigh flap with a vascularized iliac bone or fibula [12-14], reported originally by us, is an example. However, the underlying premise in all of these flap constructs is that they are essentially 'flow-through' flaps (vascularly in-series). These combinations have been noted to have an increased risk of distal flap necrosis [15] as well as prolong the operating time necessary to harvest flaps from different donor sites in cases with very long and narrow defects. In this regard, the multi-stage combined flaps are ideal, as stratifying the overall procedure into modular components allows for shorter procedures at each stage.

The name 'Orochi' is derived from a mythological Japanese eight-headed snake, akin to a 'hydra'. The term alludes to the creation of parallel vascular systems (flaps), but are connected to a single or several source vessels (Figure 3). This preserves the source vessel particularly important, e.g., in the case of the femoral vessels to the lower limb, while also minimising the number of critical microvascular anastomoses. This improves distal flap perfusion, obviates the need for the distal free-flap to be dependent on a flow-through flap, and is more physiologically in line with tissue vasculature (vascularly in parallel). 
This is demonstrated here by the two or three-staged procedure using a free TAP flap followed by a sequential triple TAP-ICAP-SCIP mega-flap (Case 2, additionally supercharged) and free SCIP flap (Case 1), both of which are in parallel to the main source vessel and independent of one another. The additional advantages are (1) shorter procedures, (2) the ability to modularly reconstruct defects up to $50 \mathrm{~cm}$ without constant intra-op positional changes, and (3) minimal donor site morbidity, i.e., a secondary defect that is closed directly without having to sacrifice functional muscles. Nevertheless, the 'Orochi' flap system requires complicated flap elevation, microvascular expertise, and coaptation of the transected lateral femoral cutaneous nerve in certain instances [2]. It should hence be performed with much pre-op planning and meticulous dissection. In addition, this triple mega-flap is not indicated for patients who received previous axillary or groin dissection.

Regarding our multistage procedure for combined tissue transfer, it still exists that the basic advantage of the combined flap reconstruction is to cover large defects in a single-stage procedure. When we proposed the one-stage combined tissue transfer until 2000, we thought one-stage was the best. However, in the last 10 years, my initial opinion has changed with long periods of experience of lots of one-stage combined chimera flap transfers, because the one-stage combined flap transfer could not exceed beyond the standard outcome. This paper introduces the preliminary my own new concept of new style combined tissue transfers to aim aesthetic reconstruction for massive complex defects. Now, I am continuously writing series of new style multistage combined tissue transfers for future advancement for aesthetic microsurgery.

Regarding Case 2, there was a criticism that the aesthetic outcome in terms of contouring on the leg could be more consistent with a single-stage free-flap procedure using supercharging DIEP flap, avoiding a patchwork flap look. This method is more common and we also have many experiences in breast reconstruction. The actual defect in Case 2 was $80 \mathrm{~cm}$ in length. Double DIEP flaps longer than $50 \mathrm{~cm}$ seem to be difficult in oriental patients. Direct closure of donor site is impossible in $80 \mathrm{~cm}$ length. Bilateral SCIP may have the potential to extend the $80 \mathrm{~cm}$ length. Also, our triple mega-flap is new and has more potential to extend the territory in future. This may be a goal of the next step flap advancement.

The creation of parallel flap systems along an extremity defect, for example, allows its use for extra long (up to $50 \mathrm{~cm}$ ) and wide defects, especially useful in young females and children. As any subsequent flap reconstruction uses the source vessel as a hub rather sacrifices its primary function, the 'Orochi' flap concept allows any number of flaps to be added onto the vascular axis without compromising distal vascularity of both the sequential flap construct or the extremity/organ.

Regarding another 'Orochi' flap, combined sequential (ALT-SCIP, DIEPSCIP, DIEP-DIEP, SCIP-SCIP, TAP-DIEP flaps etc.) and additional free-flapsare proposed. The indications of these flaps are defects with difficult repair with one-stage chimera transfer with respect to fewer techniques, manpower, and/ or surgical time; multistage tissue transfer for extensive 3-dimensional complex defects including longer and wider defects, not only in the limbs, but the whole body.

\section{CONCLUSION}

We believe that the concept and technique of the multi-stage 'Orochi' combined flaps (sequential flaps in parallel) exemplified here would open a new vista in reconstructive microsurgery.

\section{ARTICLE INFORMATION}

*Correspondence: Isao Koshima, MD, International Center for Lymphedema, Hiroshima University Hospital, 1-2-3, Kasumi, Minami-ku, Hiroshima City 734-8551, Japan. E-mail: koushimaipla@gmail.com
Received: Oct. 08, 2018; Accepted: Dec. 24, 2018; Published: Mar. 20, 2019

DOI: $10.24983 /$ scitemed.imj.2019.00108

Acknowledgements: The authors wish to thank Professor Rryukiti Kawamura, Chief of the Department of Dermatology of Yamanashi University in Japan for his support in this study.

Disclosures: The work was presented in part at the Japanese Society of Reconstructive Microsurgery in Niigata, Japan on November 11, 2011 as well as the Maliniac Lecture in the American Society of Plastic Surgery in Chicago, USA on October 13, 2014.

Ethics Approval and Consent to Participate: The study is in accordance with the ethical standards of the 1964 Helsinki declaration and its later amendments or comparable ethical standards.

Funding: The study did not receive any specific grant from funding agencies in the public, commercial, or not-for-profit sectors.

Conflict of Interest: The authors report no financial or other conflict of interest relevant to this article, which is the intellectual property of the authors.

Copyright @ 2019 The Author (s). This is an open-access article distributed under the terms of the Creative Commons Attribution 4.0 International License (CC-BY).

\section{REFERENCES}

1. Harii K, Iwaya T, Kawaguchi N. Combination myocutaneous flap and microvascular free flap. Plast Reconstr Surg 1981;68(5):700-711.

2. Gunji Y, Ariga T, Ono I. An electrical injury resulting in an extensive soft tissue defect of the chest wall: a case report.Jpn J Plast Reconstr Surg 1992;35:1135-1141.

3. Koshima I, Nanba Y, Tsutsui T, et al. Superficial circumflex iliac artery perforator flap for reconstruction of limb defects. Plast Reconstr Surg 2004;113(1):233-240.

4. Ishii CH,Jr., BostwickJ, 3rd, Raine TJ, Coleman ل, 3rd, Hester TR. Double-pedicle transverse rectus abdominis myocutaneous flap for unilateral breast and chest-wall reconstruction. Plast Reconstr Surg 1985;76(6):901-907.

5. Harashina T, Sone K, Inoue T, Fukuzumi S, Enomoto K. Augmentation of circulation of pedicled transverse rectus abdominis musculocutaneous flaps by microvascular surgery. BrJ Plast Surg 1987;40(4):367-370.

6. Takayanagi S, Ohtsuka M. Extended transverse rectus abdominis musculocutaneous flap. Plast Reconstr Surg 1989;83(6):1057-1060.

7. Core GB, Weimar R, Meland NB. The turbo gracilis myocutaneous flap. J Reconstr Microsurg 1992;8(4):267-275.

8. Koshima I, Soeda S, Ohno A. Combined tensor fasciae latae musculocutaneous flap and sartorius musculocutaneous flap for the repair of wide defects of the lower leg. Plast Reconstr Surg 1990;86(6):1198-1201.

9. Koshima I, Yamamoto H, Moriguchi T, Orita Y. Extended anterior thigh flaps for repair of massive cervical defects involving pharyngoesophagus and skin: An introduction to the "mosaic" flap principle. Ann Plast Surg 1994;32(3):321-327.

10. Koshima I. Classification of free combined or connected tissue (flap) transfers. Ann Plast Surg 1994;33:462.

11. Koshima I. A new classification of free combined or connected tissue transfers: Introduction to the concept of bridge, siamese, chimeric, mosaic, and chain-circle flaps. Acta Med Okayama 2001;55(6):329-332.

12. Hallock GG. Simultaneous transposition of anterior thigh muscle and fascia flaps: An introduction to the chimera flap principle. Ann Plast Surg 1991;27(2):126-131.

13. Koshima I, Yamamoto H, Hosoda M, Moriguchi T, Orita Y, Nagayama H. Free combined composite flaps using the lateral circumflex femoral system for repair of massive defects of the head and neck regions: An introduction to the chimeric flap principle. Plast Reconstr Surg 1993;92(3):411-420.

14. Koshima I, Hosoda S, Inagawa K, Urushibara K, Moriguchi T. Free combined anterolateral thigh flap and vascularized fibula for wide, through-and-through oromandibular defects. J Reconstr Microsurg 1998;14(8):529-534.

15. Wei FC, Celik N, Chen HC, Cheng MH, Huang WC. Combined anterolateral thigh flap and vascularized fibula osteoseptocutaneous flap in reconstruction of extensive composite mandibular defects. Plast Reconstr Surg 2002;109(1):45-52.

16. Hallock GG. Partial failure of a free flap salvaged by using the surviving portion as a "bridge" flap for revascularizing a second free flap. Plast Reconstr Surg 1997;100(4):981985. 